\title{
Thermodynamic stability of asymptotically anti-de Sitter rotating black holes in higher dimensions
}

\author{
Brian P. Dolan \\ Department of Mathematics, Heriot-Watt University \\ Edinburgh, EH14 4AS, U.K.
}

Maxwell Institute for Mathematical Sciences, Edinburgh, U.K.

Email: B.P.Dolan@hw.ac.uk

July 25, 2014 


\begin{abstract}
Conditions for thermodynamic stability of asymptotically anti-de Sitter rotating black holes in $D$-dimensions are determined. Local thermodynamic stability requires not only positivity conditions on the specific heat and the moment of inertia tensor but it is also necessary that the adiabatic compressibility be positive. It is shown that, in the absence of a cosmological constant, neither rotation nor charge is sufficient to ensure full local thermodynamic stability of a black hole.

Thermodynamic stability properties of anti-de Sitter Myers-Perry black holes are investigated for both singly spinning and multi-spinning black holes. Simple expressions are obtained for the specific heat and moment of inertia tensor in any dimension. An analytic expression is obtained for the boundary of the region of parameter space in which such space-times are thermodynamically stable.
\end{abstract}

PACS nos: 04.50.Gh; 04.60.-m; 04.70.Dy 


\section{Introduction}

Ever since Hawking's discovery [1] that black holes have a temperature associated with them, and will radiate thermal energy when isolated, the fascinating topic of black hole thermodynamics has been a major focus of research. Schwarzschild black holes have a negative specific heat and are therefore thermodynamically unstable but, for anything other than very small mass black holes, the life-time is so large that the black hole persists for time scales greater than or of the order of the age of the Universe and thermodynamic concepts can still be applied. It is possible to make a black hole thermodynamically stable by introducing a negative cosmological constant $\Lambda$ and considering asymptotically anti-de Sitter (AdS) black holes, provided the magnitude of $\Lambda$ is large enough, [2] 1]

The specific heat for an asymptotically flat black holes can also be rendered positive by rotating the black hole or by giving it an electric charge, but this does not in itself ensure full thermodynamic stability as the moment of inertia can, and indeed does, become negative. This phenomenon persists to space-times of higher dimension [4]. In dimension $D>4$ there is more than one angular momentum and the moment of inertia is a tensor but this tensor develops a negative eigenvalue in any region of parameter space for which the corresponding specific heat is positive [4], [5].

The situation for asymptotically AdS black holes in $D$ dimensions is somewhat different and, with the current interest in the AdS/CFT correspondence [6], such space-times are of considerable importance. The literature here is not as comprehensive as for the asymptotically de Sitter case, though there has been significant progress in specific cases [7]-[10]. In this paper a full description is given of the local thermodynamic stability properties of asymptotically AdS black holes in any dimension. It is shown in $\$ 2$ that all isolated black holes suffer from a local thermodynamic instability unless a negative cosmological constant is introduced. In 33 a well known class of rotating asymptotically AdS black holes, asymptotically AdS Myers-Perry black holes, is studied in detail and the thermodynamically stable region of parameter space is mapped out for all space-time dimensions. Finally $₫ 4$ summarises our results.

\footnotetext{
${ }^{1}$ They can also be stabilised by putting them in a finite volume cavity, [3].
} 


\section{General criteria for stability}

When a cosmological constant is present it was argued in [10] that the ADM mass of a black hole should be viewed, in a thermodynamic context, as the enthalpy $H$ of the thermodynamic system, rather than the heretofore more common interpretation of internal energy $U$, and this will be the philosophy adopted here. The natural thermodynamic control parameters for the enthalpy are entropy $S$, angular momentum $J$, pressure $P$ and electric charge $Q$, so we write

$$
M=H(J, S, P, Q) .
$$

The thermal energy $U(J, S, V, Q)$, which depends not only on $S, J, Q$ but also on the volume $V$, is the Legendre transform of the enthalpy

$$
U=H-P V .
$$

There are subtleties associated with the definition of the volume of a black hole, [11 - 14], but thermodynamically volume can be defined as the variable thermodynamically conjugate to the pressure [10, 15],

$$
V=\left.\frac{\partial M}{\partial P}\right|_{J, S, Q},
$$

where the pressure is produced by the cosmological constant

$$
P=-\frac{\Lambda}{8 \pi} \text {. }
$$

The introduction of a thermodynamic pressure and volume in black hole physics has generated a lot of activity on the black hole equation of state recently, for a review of the current situation see [16].

Local thermodynamic stability requires that the internal energy $U$ be a convex function [17. Equivalently the Legendre transform of $U$,

$$
\mathcal{E}(\Omega, T, P, \Phi):=U-\Omega_{i} J^{i}-T S+P V-\Phi Q=M-\Omega_{i} J^{i}-T S-\Phi Q,
$$

should be a concave function 2 We shall refer to $\left(J^{i}, S, V, Q\right)$ as extensive variables and $\left(\Omega_{i}, T, P, \Phi\right)$ as intensive variables. This classification is motivated by the canonical dimensions of these variables, as the dimensions of

\footnotetext{
${ }^{2}$ In $D$ space-time dimensions there is more than one angular momentum, labelled by the index $i$ which runs from 1 up to $N$, where $N$ is the dimension of the Cartan sub-algebra of $S O(D-1)$.
} 
$(J, S, V, Q)$ depend on $D$ while those of $\left(\Omega_{i}, T, P, \Phi\right)$ do not, as shown in the table below (with Newton's constant and the speed of light set to unity),

\begin{tabular}{|l|c|}
\hline Thermodynamic Variable & Dimension \\
\hline Mass, $M$ & $D-3$ \\
Entropy, $S$ (area) & $D-2$ \\
Angular momenta, $J^{i}$ & $D-2$ \\
Volume, $V$ & $D-1$ \\
Electric Charge, $Q$ & $D-3$ \\
Temperature, $T$ & -1 \\
Angular velocity, $\Omega_{i}$ & -1 \\
Pressure, $P(\Lambda)$ & -2 \\
Electric potential, $\Phi$ & 0 \\
\hline
\end{tabular}

Let

$$
x_{A}=\frac{\partial U}{\partial X^{A}}=\left(\Omega_{i}, T,-P, \Phi\right),
$$

with $A=1, \ldots, N+3$, denote the intensive variables and

$$
X^{A}=-\frac{\partial \mathcal{E}}{\partial x_{A}}=\left(J^{i}, S, V, Q\right)
$$

denote the extensive variables.

In the grand canonical ensemble the volume is usually kept fixed and the other thermodynamic control parameters are the intensive ones. In ordinary thermodynamics scaling arguments would imply that Legendre transforming to make all control parameters intensive results in a vanishing thermodynamic potential, $\mathcal{E}=0$, [17] (this is essentially the content of the GibbsDuhem relation). For black holes however this is not the case and $\mathcal{E}\left(x_{A}\right)$ is not trivial, in fact $\mathcal{E}$ is related to the Euclidean action $I_{E}$ by [18]

$$
\mathcal{E}=T I_{E} .
$$

We shall call the ensemble with thermodynamic potential $\mathcal{E}$ the extended canonical ensemble and $\mathcal{E}$ the extended free energy.

For asymptotically flat black holes $P=0$ and the grand canonical and extended canonical ensembles coincide, but for asymptotically AdS black holes they do not. (The relation between the microcanonical, the canonical and the grand canonical ensembles for asymptotically flat black holes was elucidated in [5].) 
A full analysis of local thermodynamic stability requires determining the region of parameter space in which all of the the eigenvalues of the Hessians, either

or

$$
\mathcal{W}_{A B}=\frac{\partial^{2} U}{\partial X^{A} \partial X^{B}}
$$

$$
\mathcal{K}^{A B}=-\frac{\partial^{2} \mathcal{E}}{\partial x_{A} \partial x_{B}},
$$

are positive. It does not matter which one is used, since it is a standard fact of Legendre transforms that $\mathcal{K}=\mathcal{W}^{-1}$, we shall start by focusing on $\mathcal{W}_{A B}$.

It was shown in [4 that all asymptotically flat, electrically neutral black holes are unstable. We present here a different derivation of this result which has the advantage of generalising it to include electric charge. Using the above dimensions in the Smarr relation [10, 19] gives

$$
(D-3) M=(D-2) \boldsymbol{\Omega} . \mathbf{J}+(D-2) T S-2 P V+(D-3) Q \Phi .
$$

With $M=U+P V$ this can be re-arranged, for $D \geq 4$, as

$$
\begin{aligned}
U & =\frac{(D-2)}{(D-3)} \Omega \cdot \mathbf{J}+\frac{(D-2)}{(D-3)} T S-\frac{(D-1)}{(D-3)} P V+Q \Phi \\
& =\sum_{B} c_{B} X^{B} x_{B},
\end{aligned}
$$

with $c_{A}$ the appropriate $D$-dependent constants. Differentiating this

$$
\begin{aligned}
x_{A}=\frac{\partial U}{\partial X^{A}} & =c_{A} x_{A}+\sum_{B} c_{B} \mathcal{W}_{A B} X^{B} \\
\Rightarrow \quad \sum_{B} \mathcal{W}_{A B}\left(c_{B} X^{B}\right) & =\left(1-c_{A}\right) x_{A},
\end{aligned}
$$

(there is no sum over $A$ here, for the rest of this section we suppress the summation convention and explicitly show all summations). The vector on $X$-space with components $\mathcal{D}^{A}=c_{A} X^{A}$ essentially represents the response of the system to a re-scaling. Using (13) we can construct the quadratic form

$$
\begin{aligned}
\sum_{A, B} \mathcal{W}_{A B} \mathcal{D}^{A} \mathcal{D}^{B} & =\sum_{A} c_{A}\left(1-c_{A}\right) x_{A} X^{A} \\
& =\frac{(D-2)}{(D-3)^{2}}\left(-\boldsymbol{\Omega} . \mathbf{J}-T S+2 \frac{(D-1)}{(D-2)} P V\right) .
\end{aligned}
$$


Clearly $\sum_{A B} \mathcal{W}_{A B} \mathcal{D}^{A} \mathcal{D}^{B}<0$ if $P=0$, heralding a thermodynamic instability for any asymptotically flat black hole in $D>3$ space-time dimensions, regardless of charge or rotation. However, provided $V>0$, a positive $P$ (negative $\Lambda$ ) can remove this particular instability if the $P V$ term is of sufficient magnitude to outweigh the $T S$ and $\boldsymbol{\Omega}$.J terms on the right-hand side of (14) 3 This is a very powerful result, it shows that the only way of making an isolated black hole locally thermodynamical stable, without imposing constraints by fixing variables, is to introduce a positive pressure. Rotation and/or electric charge alone cannot do the job unless they are fixed and not allowed to vary 4 but with no constraints there cannot be local thermodynamical stability unless $P>0$. The case $P=Q=0$ was explored in [5], and the $P>0$ scenario will be analysed in some detail in this work, but still with $Q=0$.

It is shown in appendix $\AA$ that, for $Q=0$, the mathematical requirement of positivity of $\mathcal{W}$ is equivalent to following three perfectly reasonable physical statements (assuming that the temperature and the thermodynamic volume are positive):

- the specific heat at constant $\Omega_{i}$ is positive,

$$
C_{\Omega}=\left.T \frac{\partial S}{\partial T}\right|_{\Omega, P}>0
$$

- the isentropic moment of inertia tensor

$$
\mathcal{I}^{i j}=\left.\frac{\partial J^{i}}{\partial \Omega_{j}}\right|_{S, P}=\left.\frac{\partial J^{j}}{\partial \Omega_{i}}\right|_{S, P}
$$

is a positive matrix;

\footnotetext{
${ }^{3}$ The quadratic form $\mathcal{W}_{A B}$ can be interpreted as a metric on configuration space, the Ruppeiner metric, [20], and stability requires that this metric be positive definite, or at least non-negative. In ordinary thermodynamics, the Gibbs-Duhem relation implies $c_{A}=1$, for all $A$, and $\mathcal{D}^{A}$ is always a null vector, indicating a direction of neutral stability.

${ }^{4}$ Stabilising the black hole by fixing $J$ and/or $Q$ can lead to very interesting phenomena, for example there is a phase transition between large and small black holes when $Q$ is fixed 21 or when $J$ is fixed 22. The equation of state leads to van der Waals type critical points, with mean field exponents [23, 24] and there can even be a triple point 25] and re-entrant phase transitions [26].
} 
- the adiabatic compressibility is positive,

$$
\kappa=-\left.\frac{1}{V} \frac{\partial V}{\partial P}\right|_{J^{i}, S}>0 .
$$

All perfectly reasonable physical requirements.

In the next section we shall examine these conditions in the specific case of asymptotically AdS Myers-Perry black holes in $D$-dimensions and determine the region of parameter space for which these space-times are thermodynamically stable.

\section{Asymptotically anti-de Sitter Myers-Perry black holes.}

\subsection{The metric and thermodynamic variables}

Rotating black holes in $D$-dimensions must be treated slightly differently for even and odd $D$ because the rotation group $S O(D-1)$, acting on the event horizon which is assumed to have the topology of a $(D-2)$-dimensional sphere, has different characterisations of angular momenta in the even and odd dimensional cases. The Cartan sub-algebra has dimension $\frac{D-2}{2}$ for even $D$ and $\frac{D-1}{2}$ for odd $D$ so a general state of rotation is specified by $\frac{D-2}{2}$ independent angular momenta in even $D$ and $\frac{D-1}{2}$ in odd $D$. Let $N=\left\lfloor\frac{D-1}{2}\right\rfloor$, the integral part of $\frac{D-1}{2}$, be the dimension of the Cartan sub-algebra of $S O(D-1)$, then there are $N$ independent angular momenta $J^{i}, i=1, \ldots, N$. It is notationally convenient to define $\epsilon=\frac{1+(-1)^{D}}{2}$, so $\epsilon=1$ for even $D$ and $\epsilon=0$ for odd $D$, and then

$$
N=\frac{D-1-\epsilon}{2} .
$$

In this notation the unit $(D-2)$-dimensional sphere can be described in terms of Cartesian co-ordinates $\mathbf{x}_{\alpha}$ in $\mathbf{R}^{D-1}$ by

$$
\sum_{\alpha=1}^{D-1} \mathrm{x}_{\alpha}^{2}=1,
$$


and we write this as

$$
\sum_{i=1}^{N} \rho_{i}^{2}+\epsilon y^{2}=1,
$$

where $\mathrm{x}_{2 i-1}+i \mathrm{x}_{2 i}=\rho_{i} e^{i \phi_{i}}, i=1, \ldots, N$, are complex co-ordinates for both the even and odd cases while $y=\mathrm{x}_{D-1}$ is only necessary for even $D . \rho_{i}, \phi_{i}$ and $y$ are then (over complete) co-ordinates that can be used to parameterise the $(D-2)$-sphere and, for the black hole, $J^{i}$ are angular momenta in the $\left(\mathrm{x}_{2 i-1}, \mathrm{x}_{2 i}\right)$-plane.

The first rotating black solutions to Einstein's equations in dimension greater than four were the asymptotically flat solutions of Myers and Perry [27]. Rotating black holes in 5-dimensions with a cosmological constant, $\Lambda$, were constructed in [28] and the generalisation to the $D$-dimensional metric was found in [29]: these are solutions of Einstein's equations with Ricci tensor

$$
R_{\mu \nu}=\frac{2 \Lambda}{(D-2)} g_{\mu \nu}
$$

We shall focus on $\Lambda \leq 0$ here, as the thermodynamics is then better understood, and for notational convenience we define

$$
\lambda=-\frac{2 \Lambda}{(D-1)(D-2)} \geq 0 .
$$

The line element in 29] can then be expressed, in Boyer-Linquist co-ordinates, as 5

$$
\begin{aligned}
d s^{2}= & -W\left(1+\lambda r^{2}\right) d t^{2}+\frac{2 \mu}{U}\left(W d t-\sum_{i=1}^{N} \frac{a_{i} \rho_{i}^{2} d \phi_{i}}{\Xi_{i}}\right)^{2} \\
& +\left(\frac{U}{Z-2 \mu}\right) d r^{2}+\epsilon r^{2} d y^{2}+\sum_{i=1}^{N}\left(\frac{r^{2}+a_{i}^{2}}{\Xi_{i}}\right)\left(d \rho_{i}^{2}+\rho_{i}^{2} d \phi_{i}^{2}\right) \\
& -\frac{\lambda}{W\left(1+\lambda r^{2}\right)}\left(\sum_{i=1}^{N}\left(\frac{r^{2}+a_{i}^{2}}{\Xi_{i}}\right) \rho_{i} d \rho_{i}+\epsilon r^{2} y d y\right)^{2}
\end{aligned}
$$

where

$$
\Xi_{i}=1-\lambda a_{i}^{2}
$$

${ }^{5}$ The form given here differs slightly from that in [29] in that our ordinates, $t$ and $\phi_{i}$, are related to those of [29], $\tau$ and $\varphi_{i}$, by $d \tau=d t$ and $d \phi_{i}=d \varphi_{i}-\lambda a_{i} d t$. 
and the functions $W, Z$ and $U$ are

$$
\begin{aligned}
W & =\epsilon y^{2}+\sum_{i=1}^{N} \frac{\rho_{i}^{2}}{\Xi_{i}} \\
Z & =\frac{\left(1+\lambda r^{2}\right)}{r^{2-\epsilon}} \prod_{i=1}^{N}\left(r^{2}+a_{i}^{2}\right) \\
U & =\frac{Z}{1+\lambda r^{2}}\left(1-\sum_{i=1}^{N} \frac{a_{i}^{2} \rho_{i}^{2}}{r^{2}+a_{i}^{2}}\right) .
\end{aligned}
$$

The $a_{i}$ are rotation parameters in the $\left(\mathrm{x}_{2 i-1}, \mathrm{x}_{2 i}\right)$-plane, restricted to

$$
a_{i}^{2}<1 / \lambda,
$$

and $\mu$ is a mass parameter.

Many of the properties of the space-time with line element (24) were described in [29]. There is an event horizon at $r_{h}$, the largest root of $Z-2 \mu=$ 0 , so

$$
\mu=\frac{\left(1+\lambda r_{h}^{2}\right)}{2 r_{h}^{2-\epsilon}} \prod_{i=1}^{N}\left(r_{h}^{2}+a_{i}^{2}\right)
$$

with area

$$
\mathcal{A}_{h}=\frac{\varpi}{r_{h}^{1-\epsilon}} \prod_{i=1}^{N} \frac{r_{h}^{2}+a_{i}^{2}}{\Xi_{i}},
$$

where $\varpi$ is is the volume of the round unit $(D-2)$-sphere,

$$
\varpi=\frac{2 \pi^{\frac{(D-1)}{2}}}{\Gamma\left(\frac{D-1}{2}\right)} .
$$

The Bekenstein-Hawking entropy is

$$
S=\frac{\varpi}{4 r_{h}^{1-\epsilon}} \prod_{i=1}^{N} \frac{r_{h}^{2}+a_{i}^{2}}{\Xi_{i}}
$$

and the Hawking temperature is, with $\hbar=1$,

$$
T=\frac{r_{h}}{2 \pi}\left(1+\lambda r_{h}^{2}\right) \sum_{i=1}^{N} \frac{1}{r_{h}^{2}+a_{i}^{2}}+\frac{(2-\epsilon)\left(\epsilon \lambda r_{h}^{2}-1\right)}{4 \pi r_{h}} .
$$


The angular momenta and the ADM mass, $M$, of the black hole are related to the metric parameters via

$$
\begin{aligned}
J_{i} & =\frac{\mu \varpi a_{i}}{4 \pi \Xi_{i} \prod_{j} \Xi_{j}}, \\
M & =\frac{\mu \varpi}{8 \pi \prod_{j} \Xi_{j}}\left(D-2+2 \lambda \sum_{i=1}^{N} \frac{a_{i}^{2}}{\Xi_{i}}\right) \\
& =\frac{S}{4 \pi r_{h}}(D-2)\left(1+\lambda r_{h}^{2}\right)+\lambda \sum_{i=1}^{N} J_{i} a_{i},
\end{aligned}
$$

while the angular velocities are

$$
\Omega_{i}=\frac{\left(1+\lambda r_{h}^{2}\right) a_{i}}{\left(r_{h}^{2}+a_{i}^{2}\right)} .
$$

\subsection{Thermodynamic potentials}

Following [10] the ADM mass of the black hole (33) is identified with the enthalpy

$$
M=H(J, S, P) .
$$

The Legendre transform to purely intensive variables, the extended free energy

$$
\mathcal{E}(\Omega, T, P)=H-T S-\mathbf{\Omega} . \mathbf{J}=U+P V-T S-\mathbf{\Omega} . \mathbf{J},
$$

then has a simple form for asymptotically AdS Myers-Perry black holes.

$$
\mathcal{E}=\frac{S}{4 \pi r_{h}}\left(1-\lambda r_{h}^{2}\right)
$$

The observation that this is negative for $\lambda r_{h}^{2}>1$, and hence less than the corresponding potential for pure AdS space-time with $S=0$ and no black hole, is the origin of the Hawking-Page phase transition [2].

Using the Smarr relation (111) one finds that, for any electrically neutral black hole, the internal energy, $U=M-P V$, is related to the Euclidean action $\mathcal{E}=M-T S-\Omega . J$

$$
U=\frac{(D-2) \mathcal{E}+M}{2},
$$

and this is easily checked explicitly for AdS Myers-Perry solutions. 


\subsection{Thermodynamic volume}

The thermodynamic volume, $V$, is defined as the variable thermodynamically conjugate to $P$,

$$
V=\left.\frac{\partial M}{\partial P}\right|_{S, J}=\left.\frac{16 \pi}{(D-1)(D-2)} \frac{\partial M}{\partial \lambda}\right|_{S, J} .
$$

This was evaluated for electrically neutral asymptotically AdS Myers-Perry black holes in [31], using the Smarr relation (111) with $Q=0$, and the expressions given in 33.1 for $M, T, S, \Omega_{i}$ and $J^{i}$. Alternatively one can use (39) directly, [5]. In either case the result is

$$
\begin{aligned}
V & =\frac{r_{h} \mathcal{A}_{h}}{D-1}\left\{1+\frac{\left(1+\lambda r_{h}^{2}\right)}{(D-2) r_{h}^{2}} \sum_{i=1}^{N} \frac{a_{i}^{2}}{\Xi_{i}}\right\} \\
& =\frac{r_{h} \mathcal{A}_{h}}{D-1}+\frac{8 \pi}{(D-2)(D-1)} \sum_{i=1}^{N} a_{i} J_{i} .
\end{aligned}
$$

Clearly $V>0$, since $a_{i}$ and $J^{i}$ are always of the same sign.

With the substitution $\lambda \rightarrow-g^{2}$ (40) also agrees with the black hole thermodynamic volume quoted in [32] for $\Lambda>0$, again determined by assuming the Smarr relation holds.

\subsection{Temperature}

To discuss the various other thermodynamic quantities it will prove useful to work with dimensionless variables

$$
\widetilde{a}_{i}=\frac{a_{i}}{r_{h}} \quad \text { and } \quad \tilde{\lambda}=\lambda r_{h}^{2}
$$

in terms of which the Hawking temperature (31) can be re-expressed as

$$
T=\frac{D-3-2 \Sigma_{1}+\widetilde{\lambda}\left(D-1-2 \Sigma_{1}\right)}{4 \pi r_{h}},
$$

where $\Sigma_{1}:=\sum_{i=1}^{N} \frac{\widetilde{a}_{i}^{2}}{1+\widetilde{a}_{i}^{2}}$. There is of course a restriction on $\widetilde{a}_{i}$ and $\widetilde{\lambda}$ that defines the region of parameter space where $T>0$. In general $T$ will vanish 
on a $N$-dimensional hypersurface in $\left(\widetilde{a}_{i}, \widetilde{\lambda}\right)$-space given by the zero locus of the polynomial

$$
(D-3) \prod_{i=1}^{N}\left(1+\widetilde{a}_{i}^{2}\right)-2 \sum_{i=1}^{N} \widetilde{a}_{i}^{2} \prod_{j \neq i}\left(1+\widetilde{a}_{j}^{2}\right)+\widetilde{\lambda}\left\{(D-1) \prod_{i=1}^{N}\left(1+\widetilde{a}_{i}^{2}\right)-2 \sum_{i=1}^{N} \widetilde{a}_{i}^{2} \prod_{j \neq i}\left(1+\widetilde{a}_{j}^{2}\right)\right\} .
$$

Rather than performing the most general analysis, which would get increasingly involved for larger and larger $D$, the discussion here will be limited to special configurations in which $n$ of the $\widetilde{a}_{i}$ are non-zero and equal and the remaining $\widetilde{a}_{i}$ vanish, this will prove sufficient to gain a good understanding of the thermodynamics. So we choose

$$
\widetilde{a}_{1}=\cdots=\widetilde{a}_{n}=\widetilde{a}, \quad \widetilde{a}_{n+1}=\cdots=\widetilde{a}_{N}=0 .
$$

The case $n=1$ is called singly-spinning and the cases $n>1$ will be called multi-spinning.

In these configurations, the $T=0$ hypersurface is given by

$$
D-3+(D-3-2 n) \widetilde{a}^{2}+\left(D-1+(D-1-2 n) \widetilde{a}^{2}\right) \widetilde{\lambda}=0
$$

on which

$$
\widetilde{a}^{2}=\frac{D-3+(D-1) \tilde{\lambda}}{2 n-(D-3)+(2 n-(D-1)) \widetilde{\lambda}} .
$$

We thus see that demanding positivity of $T$ puts the following restrictions on $\widetilde{a}$ :

1. If $n \leq \frac{D-3}{2}$, no restriction on $\widetilde{a}^{2}$.

2. Even $D$, if $n=N=\frac{D-2}{2}$ and $\widetilde{\lambda}<1$, then $\widetilde{a}^{2}<\frac{D-3+(D-1) \widetilde{\lambda}}{1-\widetilde{\lambda}}$.

3. Odd $D$, if $n=N=\frac{D-1}{2}, \widetilde{a}^{2}<\frac{D-3+(D-1) \tilde{\lambda}}{2}$.

Thus positivity of $T$ is only an issue for the configurations (44) when the black hole is spinning in all possible planes and $n=N$. 


\subsection{Specific Heat}

$C_{\Omega}$ can be evaluated straightforwardly and the details are left to appendix B. Here we just quote the result,

$$
C_{\Omega}=-\frac{4 \pi r_{h} T S\left(D-2+2 \bar{\Sigma}_{1}\right)}{\left(D-3+2 \bar{\Sigma}_{1}-\widetilde{\lambda}\left(D-1+2 \bar{\Sigma}_{1}\right)\right)},
$$

where $\bar{\Sigma}_{1}:=\sum_{i=1}^{p} \frac{\widetilde{a}_{i}^{2}}{1-\widetilde{a}_{i}^{2}}$. In asymptotically flat Myers-Perry space times this reduces to the result

$$
C_{\Omega}=-\frac{16 \pi^{2} r_{h}^{2} M T\left(D-2+2 \bar{\Sigma}_{1}\right)}{(D-2)\left(D-3+2 \bar{\Sigma}_{1}\right)}
$$

in [5].

Local thermodynamic stability requires that $C_{\Omega}$ be positive and this only holds in a restricted region of the $(N+1)$-dimensional parameter space $\left(\widetilde{a}_{i}, \widetilde{\lambda}\right)$. Assuming that the metric parameter $\mu$ is chosen so that there is a black hole and $r_{h}>0$, and that the condition (26) is satisfied so that $S>0$, then, from (47),

$$
\frac{C_{\Omega}}{S} \geq 0 \Leftrightarrow \frac{\left(D-3-2 \Sigma_{1}+\widetilde{\lambda}\left(D-1-2 \Sigma_{1}\right)\right)\left(D-2+2 \bar{\Sigma}_{1}\right)}{\left(D-3+2 \bar{\Sigma}_{1}-\widetilde{\lambda}\left(D-1+2 \bar{\Sigma}_{1}\right)\right)} \leq 0 .
$$

The boundary between regions of opposite sign is the locus of points where either the numerator is zero or the denominator vanishes and $C_{\Omega}$ has a pole. Introducing the notation

$$
\begin{aligned}
& \mathcal{C}_{D, s}=(D-s) \prod_{i}^{N}\left(1+\widetilde{a}_{i}^{2}\right)-2 \sum_{k=1}^{N}\left(\widetilde{a}_{k}^{2} \prod_{i \neq k}^{N}\left(1+\widetilde{a}_{i}^{2}\right)\right) \\
& \overline{\mathcal{C}}_{D, s}=(D-s) \prod_{i}^{N}\left(1-\widetilde{a}_{i}^{2}\right)+2 \sum_{k=1}^{N}\left(\widetilde{a}_{k}^{2} \prod_{i \neq k}^{N}\left(1-\widetilde{a}_{i}^{2}\right)\right),
\end{aligned}
$$

the zeros of $C_{\Omega}$ lie on hypersurfaces characterised by

$$
\overline{\mathcal{C}}_{D, 2}=0,
$$

or

$$
\mathcal{C}_{D, 3}+\widetilde{\lambda} \mathcal{C}_{D, 1}=0
$$


(the latter is the $T=0$ hypersurface). There are poles in $C_{\Omega}$ on the hypersurface characterised by

$$
\overline{\mathcal{C}}_{D, 3}-\widetilde{\lambda} \overline{\mathcal{C}}_{D, 1}=0
$$

It is curious that the zeros of $T$ and the poles of $C_{\Omega}$ are characterised by the same polynomial but with $\widetilde{a}^{2} \rightarrow-\widetilde{a}^{2}$ and $\widetilde{\lambda} \rightarrow-\widetilde{\lambda}$.

The relevant surfaces for $D=5$ are shown in figure 1,
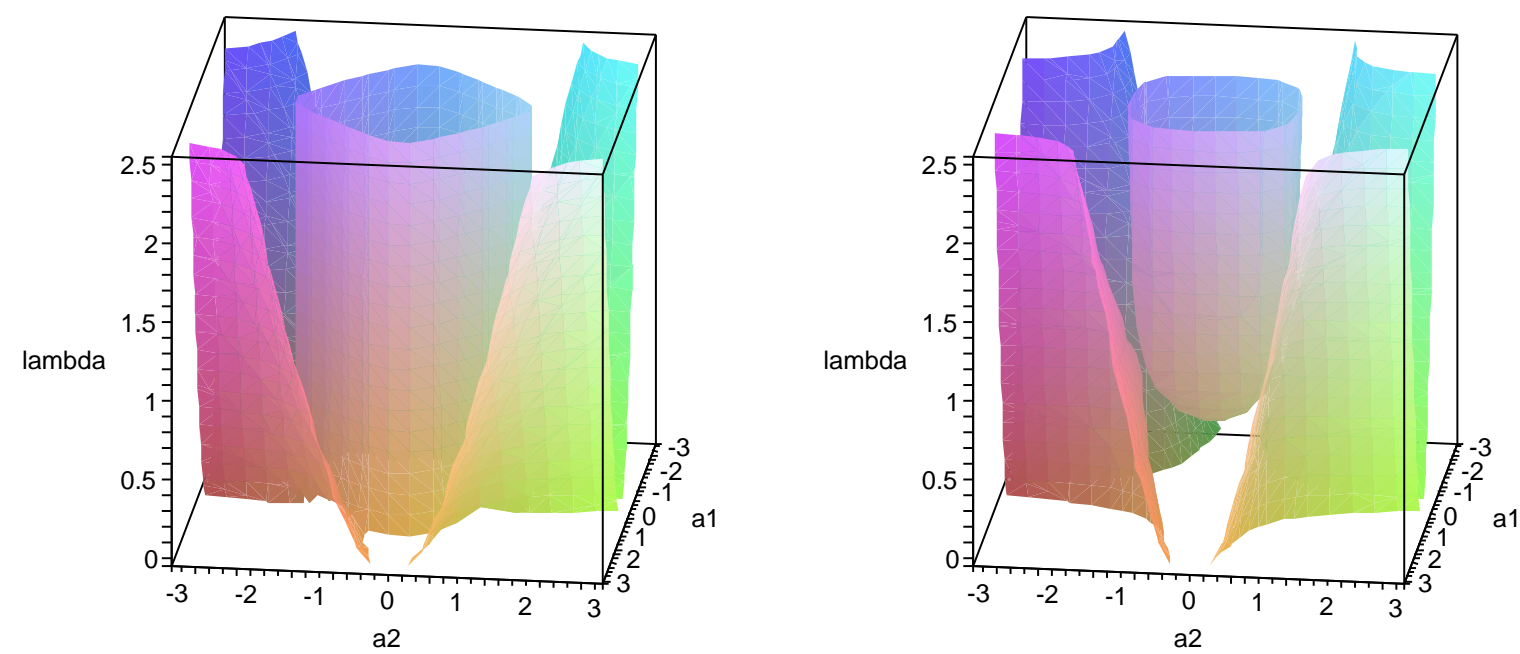

Figure 1: Surfaces on which $C_{\Omega}$ changes sign in 5-dimensions. The left-hand figure shows the locus of zeros and the right hand figure the locus of poles. The left-hand cylinder lies everywhere outside the central pillar in the righthand figure and everywhere inside the four wings of the right-hand figure, touching all the right-hand figure surfaces tangentially along the vertical lines $\widetilde{a}_{1}^{2}=\widetilde{a}_{2}^{2}=1 . C_{\Omega}$ is negative at $\widetilde{a}_{1}=\widetilde{a}_{2}=\widetilde{\lambda}=0$ and changes sign every time one of these surfaces is crossed.

For the symmetric configurations (44)

$$
\bar{\Sigma}_{1}=\frac{n \widetilde{a}^{2}}{1-\widetilde{a}^{2}}
$$

and, in the region where $T>0$, (48) requires

$$
\frac{D-2-(D-2-2 n) \widetilde{a}^{2}}{D-3-(D-1) \widetilde{\lambda}-[(D-3-2 n)-(D-1-2 n) \widetilde{\lambda}] \widetilde{a}^{2}}<0 .
$$


$C_{\Omega}$ can change sign either by passing through zero when the numerator vanishes, on the $\widetilde{\lambda}$-independent hypersurface

$$
\widetilde{a}^{2}=\frac{D-2}{D-2-2 n}
$$

or by passing through a pole when the denominator vanishes, on the hypersurface

$$
\widetilde{a}^{2}=\frac{D-3-(D-1) \tilde{\lambda}}{D-3-2 n-(D-1-2 n) \widetilde{\lambda}} .
$$

There are no singularities in the range

$$
\frac{D-3-2 n}{D-1-2 n}<\widetilde{\lambda}<\frac{D-3}{D-1}
$$

The regions in which $C_{\Omega}$ is positive are shown in figure 2. For singly spinning black holes, $n=1$, thermodynamic stability was examined in [9] and the upper left figure is essentially the same as figure 2 in that reference, expressed in slightly different variables. The analysis here extends this to multiply spinning black holes.

For $\widetilde{a}^{i}=0$ (or equivalently $n=0$ ) a negative cosmological constant of sufficient magnitude, $\widetilde{\lambda}>\frac{D-3}{D-1}$, can render $C_{\Omega}$ positive, but when $\widetilde{a}^{2}$ is increased the value of $\widetilde{\lambda}$ necessary to ensure $C_{\Omega}>0$ must also increase at first, until $\widetilde{a}^{2}=\frac{D-2}{D-2-2 n}$ (for $n \leq \frac{D-3}{2}$ ) at which point $C_{\Omega}$ becomes positive even for $\widetilde{\lambda}=0$.

The symmetric configurations (44) do not reveal all of the structure, however. In $D=5$, for example, in addition to the singular branch shown in the bottom right panel of figure 2 the denominator of $C_{\Omega}$ also vanishes for $\widetilde{a}_{1}^{2}=\widetilde{a}_{2}^{2}=1$ for any $\widetilde{\lambda}$, because there is a factor $\left(1-\widetilde{a}^{2}\right)$ in the polynomial (52). This is cancelled by a similar factor in the numerator of $C_{\Omega}$ (55) and $C_{\Omega}$ is in fact finite on $\widetilde{a}_{1}^{2}=\widetilde{a}_{2}^{2}=1$, for any $\widetilde{\lambda} \neq 1$. Away from the symmetric plane $\widetilde{a}_{1}=\widetilde{a}_{2}$ however the singular surface bifurcates and, if $\widetilde{\lambda}>\frac{1}{2}$ (or, more generally, $\widetilde{\lambda}>\frac{D-3}{D-1}$ ), there are more regions where $C_{\Omega}$ changes sign. In $D=5$, if $\widetilde{\lambda}>\frac{1}{2}$ is fixed and we come out from $\widetilde{a}_{1}=\widetilde{a}_{2}=0$ in a general direction in the $\widetilde{a}_{1}-\widetilde{a}_{2}$ plane, we start with $C_{\Omega}>0$, pass through a singularity, then a zero and then a second singularity, so $C_{\Omega}$ changes sign three times and eventually emerges with a negative sign. In the direction $\widetilde{a}_{1}=\widetilde{a}_{2}=\widetilde{a}$ however one of the singular surfaces and the zero surface meet so $C_{\Omega}$ only 

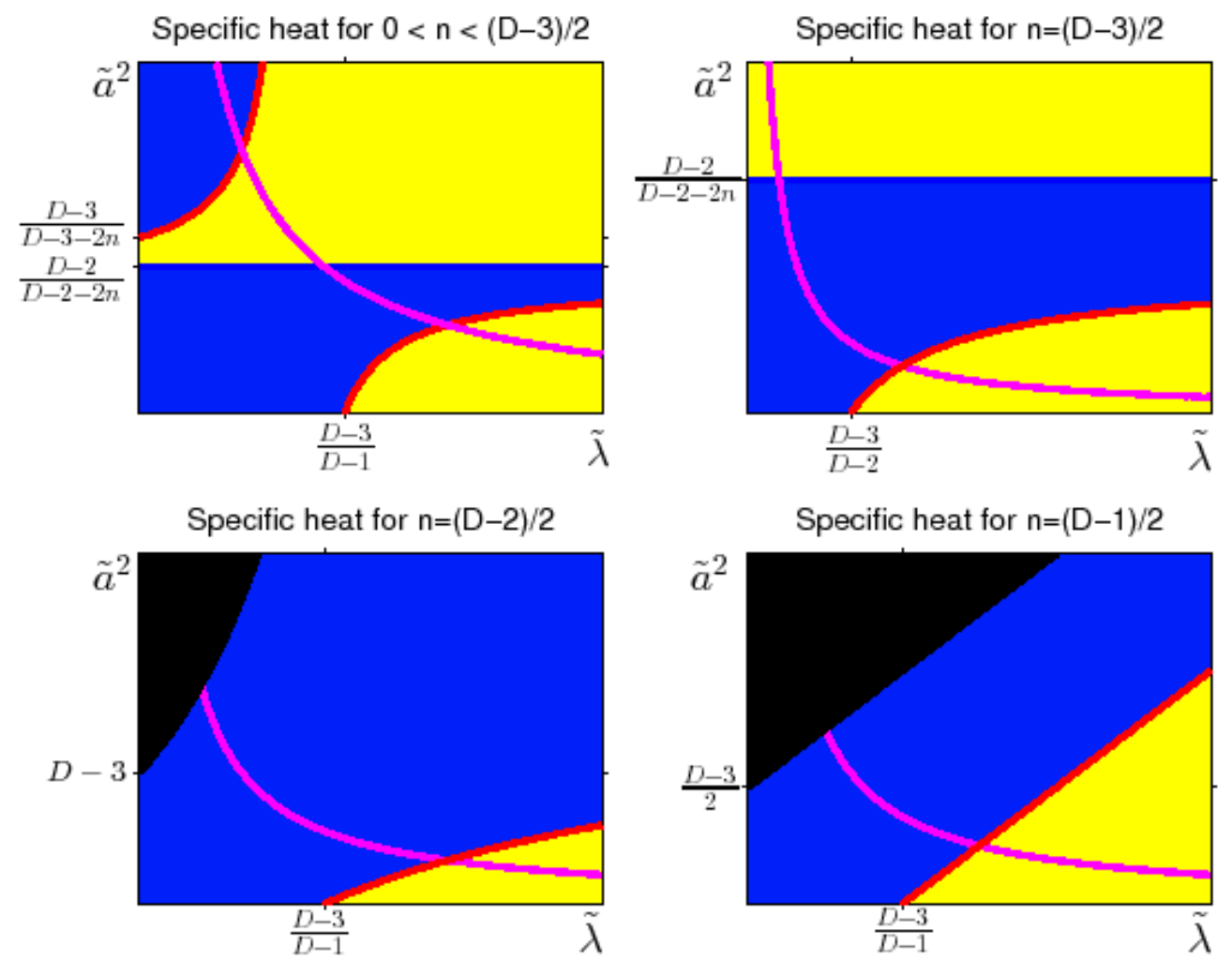

Figure 2: Regions in the $\widetilde{a}^{2}-\tilde{\lambda}$ plane where $C_{\Omega}>0$ are coloured yellow, regions where it is negative are blue (the generic case is the top-left figure, the other three panels are for special values of $n$, the black regions are where $T<0) . \quad C_{\Omega}$ is zero on the horizontal lines in the upper two figures and diverges on the red curves. The geometrical constraint (26) requires that only the area below the hyperbola $\widetilde{\lambda} \widetilde{a}^{2}=1$ (magenta) is allowed.

changes sign once, going straight from positive to negative. The full phase diagram is therefore more involved than shown in figure 2. However when the restrictions arising from positivity of the moment of inertia tensor considered in the next subsection are folded into the analysis, the symmetric configurations (44) still prove to be sufficient for a good understanding of the topology of the local thermodynamically stable region of phase space. 


\subsection{Isentropic moment of Inertia}

The isentropic moment of inertia tensor is defined as $\mathcal{I}^{i j}=\left(\frac{\partial J^{i}}{\partial \Omega_{j}}\right)_{S, P}$, which is derived in appendix C, It has the form

$$
\mathcal{I}^{i j}=\frac{r_{h} S}{2 \pi \Xi_{i} \Xi_{j}} \frac{\left(1+\widetilde{a}_{i}^{2}\right)\left(1+\widetilde{a}_{j}^{2}\right)}{\left(1-\widetilde{a}_{i}^{2}\right)\left(1-\widetilde{a}_{j}^{2}\right)}\left\{\bar{\Xi}_{i}\left(1-\widetilde{a}_{i}^{2}\right) \delta^{i j}-\frac{2(1+\widetilde{\lambda}) \widetilde{a}_{i} \widetilde{a}_{j}}{\left(D-2+2 \bar{\Sigma}_{1}\right)}\right\},
$$

where

$$
\bar{\Xi}_{i}:=1+\lambda a_{i}^{2} .
$$

The determinant of $\mathcal{I}$ is

$$
\operatorname{det} \mathcal{I}=\left(\frac{r_{h} S}{2 \pi}\right)^{N} \frac{\left(D-2-2 \widetilde{\lambda} \sum_{k} \frac{\widetilde{a}_{k}^{2}}{\bar{\Xi}_{k}}\right)}{\left(D-2+2 \bar{\Sigma}_{1}\right)} \prod_{i=1}^{N} \frac{\left(1+\widetilde{a}_{i}^{2}\right)^{2} \bar{\Xi}_{i}}{\left(1-\widetilde{a}_{i}^{2}\right) \Xi_{i}^{2}} .
$$

Hence at least one eigenvalue has a pole on the hypersurface (55), where $C_{\Omega}$ has a zero, and there are also possible poles whenever any $\widetilde{a}_{i}^{2}=1$ or $\widetilde{\lambda}_{\vec{a}}^{2}=1$, though these last are never really achieved due to the constraint (26). The same constraint shows that the determinant never vanishes, since it implies that $\sum_{k} \frac{\tilde{\lambda} \widetilde{a}_{k}^{2}}{\bar{\Xi}_{k}}<\frac{N}{2}$ so

$$
D-2-2 \widetilde{\lambda} \sum_{k} \frac{\widetilde{a}_{k}^{2}}{\bar{\Xi}_{k}}>D-2-N>0 .
$$

The location of the poles of $\operatorname{det} \mathcal{I}$ are determined purely by $\widetilde{a}_{i}$ and are independent of $\widetilde{\lambda}$, and these have already been studied for $\widetilde{\lambda}=0$ in [5].

The eigenvalues do however depend on $\widetilde{\lambda}$, even thought the locus of poles of $\operatorname{det} \mathcal{I}$ does not. In the symmetric configurations of the from (44) the moment of inertia tensor (58) has three distinct eigenvalues, stripping off the positive pre-factor $\frac{r_{h} S}{2 \pi}$ these are

$$
\begin{aligned}
& \lambda_{1}=\frac{\left(1+\widetilde{a}^{2}\right)^{2}\left(D-2+(D-2-2 n) \widetilde{\lambda} \widetilde{a}^{2}\right)}{\Xi^{2}\left(D-2-(D-2-2 n) \widetilde{a}^{2}\right)} \\
& \lambda_{2}=\frac{\left(1+\widetilde{\lambda} \widetilde{a}^{2}\right)\left(1+\widetilde{a}^{2}\right)^{2}}{\Xi^{2}\left(1-\widetilde{a}^{2}\right)}, \\
& \lambda_{3}=1,
\end{aligned}
$$


with $\lambda_{2}$ being $(n-1)$-times degenerate (and hence only present for $n \geq 2$ ) and $\lambda_{3}$ being $(N-n)$-times degenerate (and only present for $\left.n<N\right)$ ). Both $\lambda_{1}$ and $\lambda_{2}$ are positive for small $\widetilde{a}^{2}$, but $\lambda_{2}$ has a pole and changes sign at $\widetilde{a}^{2}=1$ while $\lambda_{1}$ has a pole and changes sign at $\widetilde{a}^{2}=\frac{D-2}{D-2-2 n}$.

Hence the moment of inertia tensor is positive if and only if one of the following conditions holds:

1. $n=0$;

2. $n=1$ and $0 \leq \widetilde{a}^{2}<\frac{D-2}{D-4}$ (this excludes the region above the horizontal lines in the upper two plots in figure 21);

3. $2 \leq n \leq N$, positivity of $\lambda_{2}$ requires $0 \leq \widetilde{a}^{2}<1$;

For all $1 \leq n \leq N$, positivity of the moment of inertia tensor combined with positivity of $C_{\Omega}$ effectively constrains parameter space to lie in the yellow region below the line $\widetilde{a}^{2}=1$ in figure 2 ,

\section{Conclusions}

Necessary and sufficient conditions for local thermodynamical stability of rotating black holes in asymptotically anti-de Sitter $D$-dimensional space time have been explored. In asymptotically flat space-time no amount of rotation and/or charge can completely stabilise the system, but a positive pressure, in the form of a negative cosmological constant can make black holes locally thermodynamically stable.

General conditions for local thermodynamical stability of an electrically neutral black hole are:

- the specific heat at constant $\Omega_{i}$ and $P$ is positive,

$$
C_{\Omega, P}=\left.T \frac{\partial S}{\partial T}\right|_{\Omega, P}>0
$$

- the isentropic moment of inertia tensor

$$
\mathcal{I}^{i j}=\left.\frac{\partial J^{i}}{\partial \Omega_{j}}\right|_{S, P}=\left.\frac{\partial J^{j}}{\partial \Omega_{i}}\right|_{S, P}
$$

is a positive $N \times N$ matrix, where $N$ is the rank of $S O(D-2)$; 
- the adiabatic compressibility is positive,

$$
\kappa=-\left.\frac{1}{V} \frac{\partial V}{\partial P}\right|_{J^{i}, S}>0 .
$$

$P=-\frac{\Lambda}{8 \pi}$ here is the pressure associated with the cosmological constant and the thermodynamic volume is defined by

$$
V=\left.\frac{\partial H}{\partial P}\right|_{J, S},
$$

where the enthalpy $H(J, S, P)$ is equated with the black hole ADM mass, $M$.

For electrically neutral black holes there is a simple relation between the thermal energy, $U$, the enthalpy, $M$, and the extended free energy, $\mathcal{E}$, given by equation (38).

These conditions have been analysed in detail for asymptotically AdS Myers-Perry black holes. Given the complexity of the metric the relevant thermodynamic quantities have remarkably simple expressions when expressed in terms of the dimensionless variables $\widetilde{a}^{i}=\frac{a^{i}}{r_{h}}$ and $\widetilde{\lambda}=\frac{2|\Lambda| r_{h}^{2}}{(D-1)(D-2)}$. The extended canonical potential (the Euclidean action times the temperature) has the very simple expression (37) in terms of the entropy.

For symmetric configurations of the form (44) the region of parameter space $\left(\widetilde{a}_{i}, \widetilde{\lambda}\right)$ for which such black holes are locally thermodynamically stable is the bright yellow region in the bottom right of figure 3. For asymptotically AdS Myers-Perry space-times it is already known that the adiabatic compressibility is always positive, [33]. The singularity structure of the isentropic moment of inertia is independent of $\widetilde{\lambda}$, once the constraint (26) had been taken in to account, and positivity of the eigenvalues merely demands that $\widetilde{a}_{i}^{2}<1$, except for singly spinning black holes, $n=1$, in which case this is relaxed to $\widetilde{a}^{2}<\frac{D-2}{D-4}$ (in $D=4$ the isentropic moment of inertia is always positive, though the isothermal moment of inertia is not [34]). The remaining requirements for local thermodynamic stability come from demanding positivity of the specific heat, $C_{\Omega}$.

When the condition for stability against Hawking-Page phase transition are folded in, the region of stability is restricted further to the bright yellow region in figure 3 , lying to the right of $\widetilde{\lambda}=1$.

These thermodynamic constraints must of course be supplemented by possible further constraints required to avoid the super-radiant instability of 


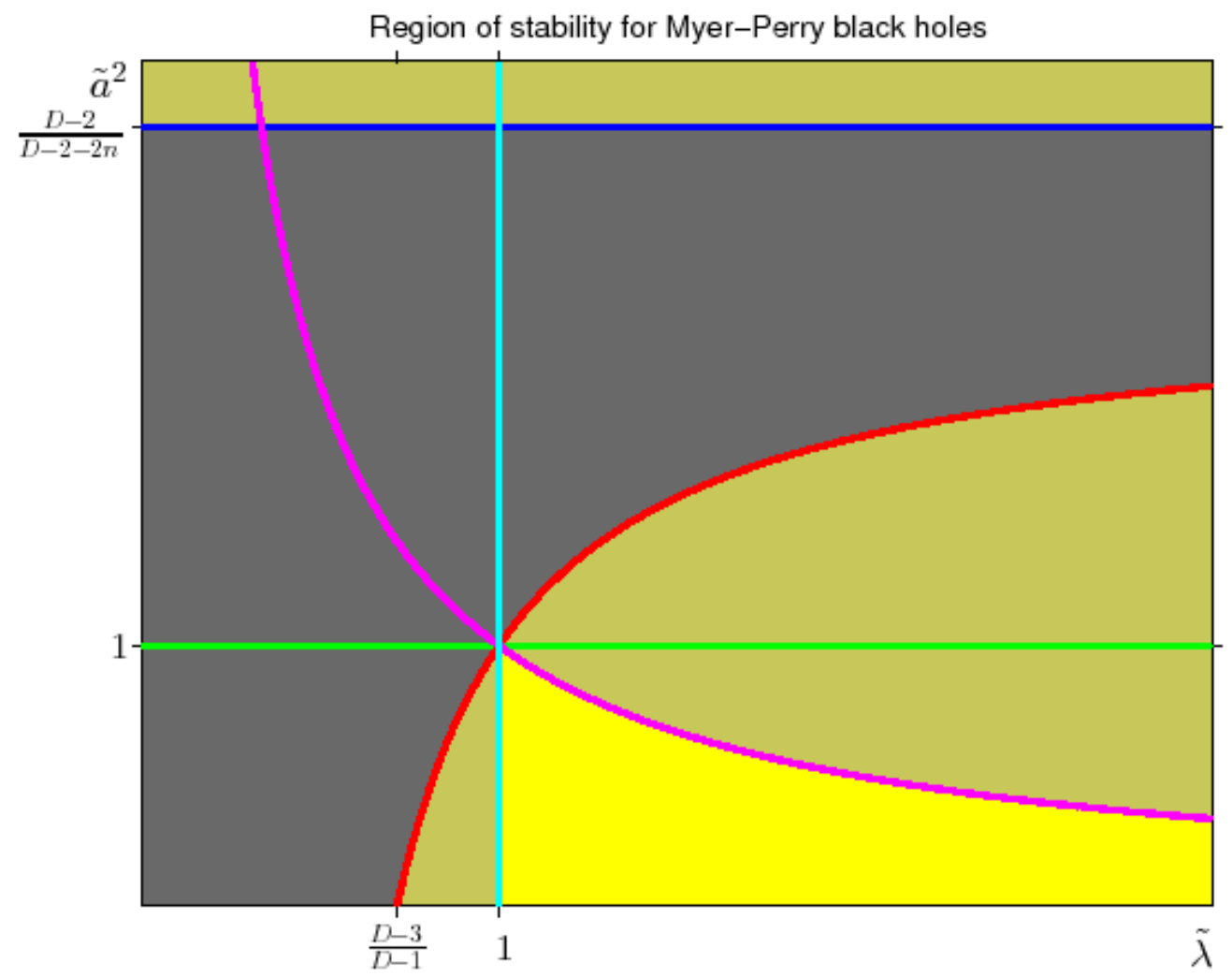

Figure 3: Region of thermodynamic stability for asymptotically AdS MyersPerry black holes, for symmetric configurations (44) with $1<n \leq \frac{D-3}{2}$. The thermodynamically stable region is the bright yellow region at the bottom right-hand part of the figure of the figure: to the left of the vertical line at $\widetilde{\lambda}=1$ the black hole is unstable against the Hawking-Page phase transition and the region above the hyperbola (magenta) is excluded by the geometric constraint (26). The isentropic moment of inertia tensor develops a negative eigenvalue above the horizontal line $\tilde{a}^{2}=1$ (green) and $C_{\Omega}$ diverges on the red curve. (For $n=1$ the horizontal green line is moved up to the horizontal blue line at $\tilde{a}^{2}=\frac{D-2}{D-4}$.) For multi-spinning black holes with $n=N$ the region of stability is the same, though some of the other curves change shape.

the classical solutions themselves, discussed in [9] and [35]-[37]. The super- 
radiant curve for $n=1$ is $\Omega^{2} \lambda=1$, which translates in our variables to

$$
\widetilde{a}^{2}=\frac{1}{\widetilde{\lambda}} \quad \text { or } \quad \widetilde{a}^{2}=\widetilde{\lambda},
$$

and the local thermodynamic stability conditions $\widetilde{a}^{2}<1 / \tilde{\lambda}$ with $\tilde{\lambda}>1$ ensure that the black hole will not super-radiate.

It would be of interest to extend this analysis to include electrically charged black holes, although from the discussion in section \$2 this cannot change the fact that such black holes are locally thermodynamically unstable for $\Lambda=0$ when unconstrained, the $\Lambda<0$ case would introduce another dimension into the phase diagram. One should also not forget that the thermodynamic stability of various constrained ensembles would be of interest, while there has already been a substantial amount of work on this for $Q \neq 0$ and $J^{i}=0$ a complete analysis including both in any $D$ is not yet available. The considerations here could also be applied to other rotating solutions in higher dimensions with event horizon topologies that differ from spheres, such as black rings and black saturns [38]-[44], although there are no exact solutions of this form currently known in the asymptotically AdS case there are approximate solutions in certain regimes of parameter space.

\section{A General stability criteria}

In this appendix we examine the criteria for thermodynamic stability for electrically neutral black holes in some detail. We first show that the Hessian (9) can be partially diagonalised by changing variables from $X^{A}=\left(J^{i}, S, V\right)$, with $A=1, \ldots, N+2$, to

$$
X^{A^{\prime}}=\left(X^{1}, \ldots, X^{N+1}, x_{N+2}\right),
$$

so, in particular, $X^{N+2^{\prime}}=x_{N+2}=\left.\frac{\partial U}{\partial V}\right|_{X^{a}}=-P$ and $X^{a^{\prime}}=X^{a}$ for $a=$ $1, \ldots, N+1$. The stability properties can equally well be explored in these co-ordinates: although the magnitude of the eigenvalues of the Hessian may change under this co-ordinate transformation, the signature does not, so $\mathcal{W}_{A B}=\frac{\partial^{2} U}{\partial X^{A} \partial X^{B}}$ is a positive matrix if and only if $\mathcal{W}_{A^{\prime} B^{\prime}}$ is, provided the co-ordinates transformation is not singular.

Let $a, b=1, \ldots N+1$ and

$$
\xi_{a}=\frac{\partial^{2} U}{\partial X^{a} \partial X^{N+2}}=\left.\frac{\partial x_{N+2}}{\partial X^{a}}\right|_{X^{N+2}}=\left.\frac{\partial x_{a}}{\partial X^{N+2}}\right|_{\vec{X}},
$$


where $\vec{X}=\left(X^{1}, \ldots, X^{N+1}\right)$. Then

$$
\mathcal{W}_{A B}=\left(\begin{array}{cc}
\mathcal{W}_{a b} & \xi_{a} \\
\xi_{b} & \mathcal{W}_{N+2, N+2}
\end{array}\right)
$$

Changing variables from $X^{A}$ to $X^{A^{\prime}}$,

$$
\frac{\partial X^{A^{\prime}}}{\partial X^{B}}=\left(\begin{array}{ccc}
\delta_{i j} & \mathbf{0} & \mathbf{0} \\
\mathbf{0}^{t} & 1 & 0 \\
\xi_{j} & \xi_{N+1} & -\left.\frac{\partial P}{\partial V}\right|_{J, S}
\end{array}\right)
$$

with $i, j=1, \ldots, N$ and $\mathbf{0}$ an $N$-component column of zeros, results in

$$
\mathcal{W}_{A B} d X^{A} d X^{B}=\mathcal{W}_{A^{\prime} B^{\prime}} d X^{A^{\prime}} d X^{B^{\prime}}
$$

where

$$
\mathcal{W}_{A^{\prime} B^{\prime}}=\left(\begin{array}{cc}
\mathcal{W}_{a b}-\left(\mathcal{W}_{N+2, N+2}\right)^{-1} \xi_{a} \xi_{b} & 0 \\
0 & \left(\mathcal{W}_{N+2, N+2}\right)^{-1}
\end{array}\right)
$$

This expression can be further simplified by noting that

$$
\begin{aligned}
\mathcal{W}_{a b}=\left.\frac{\partial x_{b}}{\partial X^{a}}\right|_{X^{N+2}} & =\left.\frac{\partial x_{b}}{\partial X^{a}}\right|_{x_{N+2}}+\left.\left.\frac{\partial x_{b}}{\partial x_{N+2}}\right|_{\vec{X}} \frac{\partial x_{N+2}}{\partial X^{a}}\right|_{X^{N+2}} \\
& =\left.\frac{\partial x_{b}}{\partial X^{a}}\right|_{x_{N+2}}+\left.\left.\left.\frac{\partial x_{b}}{\partial X^{N+2}}\right|_{\vec{X}} \frac{\partial X^{N+2}}{\partial x_{N+2}}\right|_{\vec{X}} \frac{\partial x_{N+2}}{\partial X^{a}}\right|_{X^{N+2}} \\
& =\left.\frac{\partial x_{b}}{\partial X^{a}}\right|_{x_{N+2}}+\left(\mathcal{W}_{N+2, N+2}\right)^{-1} \xi_{a} \xi_{b},
\end{aligned}
$$

thus

$$
\mathcal{W}_{a b}-\left(\mathcal{W}_{N+2, N+2}\right)^{-1} \xi_{a} \xi_{b}=\left.\frac{\partial x_{b}}{\partial X^{a}}\right|_{x^{N+2}} .
$$

Denote this $(N+1) \times(N+1)$ matrix by $\widetilde{\mathcal{W}}$, with components

$$
\widetilde{\mathcal{W}}_{a b}=\left.\frac{\partial x_{b}}{\partial X^{a}}\right|_{x^{N+2}}=\left.\frac{\partial^{2} H}{\partial X^{a} \partial X^{b}}\right|_{x_{N+2}}
$$

where $H=U+P V=M$ is the enthalpy. Then $\mathcal{W}$ is partially diagonalised in the $X^{A^{\prime}}$ co-ordinates

$$
\mathcal{W}_{A^{\prime} B^{\prime}}=\left(\begin{array}{cc}
\widetilde{\mathcal{W}}_{a b} & 0 \\
0 & \left(\mathcal{W}_{N+2, N+2}\right)^{-1}
\end{array}\right)
$$


Now

$$
\mathcal{W}_{N+2, N+2}=-\left.\frac{\partial P}{\partial V}\right|_{J^{i}, S}
$$

which is related to the adiabatic compressibility

$$
\kappa_{J, S}=-\left.\frac{1}{V} \frac{\partial V}{\partial P}\right|_{J^{i}, S}
$$

by

$$
\mathcal{W}_{N+2, N+2}=\frac{1}{\kappa_{J, S} V}
$$

so

$$
\mathcal{W}_{A^{\prime} B^{\prime}}=\left(\begin{array}{cc}
\widetilde{\mathcal{W}}_{a b} & 0 \\
0 & \kappa_{J, S} V
\end{array}\right) \text {. }
$$

Assuming that adiabatic compressibility and the volume are positive (which is known to be the case for asymptotically AdS Myers-Perry black holes in $D$-dimensions, [33]), the question of thermodynamic stability has now been reduced to the question of positivity of $\widetilde{\mathcal{W}}$. In terms of familiar thermodynamic quantities $\widetilde{\mathcal{W}}$ decomposes as

$$
\widetilde{\mathcal{W}}=\left(\begin{array}{cc}
\left(\mathcal{I}_{S}^{-1}\right)_{i j} & \zeta_{i} \\
\zeta_{j} & \frac{1}{\beta C_{J, P}}
\end{array}\right)
$$

where $\mathcal{I}_{S}^{i j}=\left.\frac{\partial J^{i}}{\partial \Omega_{j}}\right|_{S, P}$ is the isentropic moment of inertia tensor, $\beta=\frac{1}{T}, C_{\mathbf{J}, P}$ is the heat capacity at constant angular momentum and pressure and

$$
\zeta_{i}=\left.\frac{\partial T}{\partial J^{i}}\right|_{S, P}=\left.\frac{\partial \Omega_{i}}{\partial S}\right|_{J, P}
$$

(equation (82) is a Maxwell relation).

We can try to continue the process of partial diagonalisation and make a further coordinate transformation to

$$
X^{A^{\prime \prime}}=\left(X^{1}, X^{2}, \ldots, X^{N}, x_{N+1}, x_{N+2}\right)
$$

with $x_{N+1}=T, x_{N+2}=-P$ and $X^{i^{\prime \prime}}=X^{i^{\prime}}=X^{i}$ for $i=1, \ldots, N$.

$$
\frac{\partial X^{A^{\prime \prime}}}{\partial X^{B^{\prime}}}=\left(\begin{array}{ccc}
\delta_{i j} & \mathbf{0} & \mathbf{0} \\
\sigma_{j} & \sigma_{N+1} & \sigma_{N+2} \\
\mathbf{0}^{t} & 0 & 1
\end{array}\right)
$$


where

$$
\sigma_{i}=\left.\frac{\partial T}{\partial J^{i}}\right|_{S, P}=\left.\frac{\partial \Omega_{i}}{\partial S}\right|_{J, P}, \quad \sigma_{N+1}=\left.\frac{\partial T}{\partial S}\right|_{J, P} \quad \text { and } \quad \sigma_{N+2}=-\left.\frac{\partial T}{\partial P}\right|_{J, S} .
$$

In these variables

$$
\mathcal{W}_{A^{\prime \prime} B^{\prime \prime}}=\left(\begin{array}{ccc}
\widetilde{\widetilde{\mathcal{W}}}_{i j} & \mathbf{0} & \mathbf{0} \\
\mathbf{0}^{t} & \beta C_{J, P} & -\beta C_{J, P} \sigma_{N+2} \\
\mathbf{0}^{t} & -\beta C_{J, P} \sigma_{N+2} & \kappa_{J, S} V+\beta C_{J, P} \sigma_{N+2}^{2}
\end{array}\right)
$$

with

$$
\widetilde{\widetilde{\mathcal{W}}}_{i j}=\widetilde{\mathcal{W}}_{i j}-\beta C_{J, P} \sigma_{i} \sigma_{j}
$$

In fact $\widetilde{\widetilde{\mathcal{W}}}$ is the inverse of the isothermal moment of inertia tensor, $\mathcal{I}_{T}$. To see this note that

$$
\left(\mathcal{I}_{T}\right)^{i j}=\left.\frac{\partial J^{i}}{\partial \Omega_{j}}\right|_{T, P}
$$

and $\Omega_{i}\left(X^{A^{\prime \prime}}\right)=\Omega_{i}(J, T, P)$ so

$$
\begin{aligned}
\widetilde{\mathcal{W}}_{i j} & =\left.\frac{\partial \Omega_{i}}{\partial J^{j}}\right|_{S, P}=\left.\frac{\partial \Omega_{i}}{\partial J^{j}}\right|_{T, P}+\left.\left.\frac{\partial \Omega_{i}}{\partial T}\right|_{J, P} \frac{\partial T}{\partial J^{i}}\right|_{S, P} \\
& =\left.\frac{\partial \Omega_{i}}{\partial J^{j}}\right|_{T, P}+\left.\left.\left.\frac{\partial \Omega_{i}}{\partial S}\right|_{J, P} \frac{\partial S}{\partial T}\right|_{J, P} \frac{\partial T}{\partial J^{i}}\right|_{S, P} \\
& =\left(\mathcal{I}_{T}^{-1}\right)_{i j}+\beta C_{J, P} \sigma_{i} \sigma_{j},
\end{aligned}
$$

since

$$
C_{J, P}=\left.T \frac{\partial S}{\partial T}\right|_{J, P}
$$

and the result follows from (87).

So we can write $(\underline{86)}$ as

$$
\mathcal{W}_{A^{\prime \prime} B^{\prime \prime}}=\left(\begin{array}{ccc}
\left(\mathcal{I}_{T}^{-1}\right)_{i j} & \mathbf{0} & \mathbf{0} \\
\mathbf{0}^{t} & \beta C_{J, P} & -\beta C_{J, P} \sigma_{N+2} \\
\mathbf{0}^{t} & -\beta C_{J, P} \sigma_{N+2} & \kappa_{J, S} V+\beta C_{J, P} \sigma_{N+2}^{2}
\end{array}\right) .
$$

The physical conditions for complete local thermodynamic stability are now clear: the isothermal moment of inertia tensor $\mathcal{I}_{T}$ must be a positive matrix, which of course ensures that its inverse is also positive; and in addition 
the $2 \times 2$ matrix

$$
\left(\begin{array}{cc}
\beta C_{J, P} & -\beta C_{J, P} \sigma_{N+2} \\
-\beta C_{J, P} \sigma_{N+2} & \kappa_{J, S} V+\beta C_{J, P} \sigma_{N+2}^{2}
\end{array}\right)
$$

must be positive. The determinant of this matrix is just $\beta \kappa_{J, S} C_{J, P} V$, so positivity is ensured by demanding that the isentropic compressibility $\kappa_{J, S}$ is positive and that the heat capacity $C_{J, P}$ is positive.

The final conclusion is that local thermodynamic stability holds if and only if the following three perfectly reasonable conditions hold (assuming that $\beta$ and $V$ are positive):

- $\kappa_{J, S}>0$

- $C_{J, P}>0$

- $\mathcal{I}_{T}$ is a positive matrix.

At fixed pressure positivity of $C_{J}$ and $\mathcal{I}_{T}$ is equivalent to positivity of the specific heat at constant angular velocity, $C_{\Omega}$, and positivity of the isentropic moment of inertia tensor, $\mathcal{I}_{S}$. This was proven in [5] for $P=0$ and the same analysis goes through for any $P \geq 0$. The essential idea of the proof is to Legendre transform $H\left(J^{1}, \ldots, J^{N}, S, P\right)$ to

$$
G\left(\Omega_{1}, \ldots, \Omega_{N}, T, P\right)=H-T S-\Omega . J
$$

Now $\left(\mathcal{I}_{S}^{-1}\right)_{i j}=\frac{\partial^{2} H}{\partial J^{i} \partial J^{j}}$ and $\frac{1}{\beta C_{J, P}}=\frac{\partial^{2} H}{\partial S^{2}}$ are components of the Hessian of $H$ while $\left(\mathcal{I}_{T}\right)_{i j}=-\frac{\partial^{2} G}{\partial \Omega_{i} \partial \Omega_{j}}$ and $\beta C_{\Omega, P}=-\frac{\partial^{2} G}{\partial T^{2}}$ are components of the Hessian of $-G$. As matrices these Hessians are inverses of each other, so positivity of one ensures positivity of the other. Indeed the identity

$$
\beta C_{J} \operatorname{det}\left(\mathcal{I}_{T}\right)=\beta C_{\Omega} \operatorname{det}\left(\mathcal{I}_{S}\right),
$$

proven in [5] for $P=0$, also holds for $P>0$.

An equivalent set of conditions for complete local thermodynamic stability is therefore that the following conditions hold (again assuming that $\beta$ and $V$ are positive):

- $\kappa_{J, S}>0$

- $C_{\Omega, P}>0$ 
- $\mathcal{I}_{S}$ is a positive matrix.

In the text the example of asymptotically anti-de Sitter Myers-Perry black holes is treated in detail and it transpires that it is much easier to calculate $\mathcal{I}_{S}$ and $C_{\Omega, P}$ for these metrics in $D$-dimensional space-time than it is to calculate $\mathcal{I}_{T}$ and $C_{J, P}$. In the text we therefore focus on the former, in particular only $\mathcal{I}_{S}$ is considered and the subscript $S$ is dropped, $\mathcal{I}=\mathcal{I}_{S}$.

\section{B Specific heat at constant angular velocity}

The specific heat at constant $\Omega$ is straightforward to determine, using the same kind if calculations as those in [5] for the asymptotically flat case. In terms of the dimensionless quantities

$$
\widetilde{a}_{i}:=\frac{a_{i}}{r_{h}}
$$

and

$$
\Xi_{i}:=1-\lambda a_{i}^{2}=1-\widetilde{\lambda} \widetilde{a}_{i}^{2}
$$

we have

$$
\begin{gathered}
S=\frac{\varpi r_{h}^{D-2}}{4} \prod_{i=1}^{N} \frac{\left(1+\widetilde{a}_{i}^{2}\right)}{\Xi_{i}}, \\
T=\frac{1}{4 \pi r_{h}}\left\{D-3-2 \Sigma_{1}+\widetilde{\lambda}\left(D-1-2 \Sigma_{1}\right)\right\} \\
\Omega_{i}=\frac{(1+\widetilde{\lambda}) \widetilde{a}_{i}}{r_{h}\left(1+\widetilde{a}_{i}^{2}\right)} .
\end{gathered}
$$

where $\Sigma_{1}:=\sum_{i=1}^{N} \frac{\widetilde{a}_{i}^{2}}{1+\widetilde{a}_{i}^{2}}$.

The specific heat at constant angular velocity and pressure is defined as

$$
C_{\Omega}=T\left(\frac{\partial S}{\partial T}\right)_{\Omega, \lambda}
$$

From (99) we find

$$
\Omega_{i}=\left.\mathrm{const} \quad \Rightarrow \quad d \widetilde{a}_{i}\right|_{\Omega, \lambda}=\left.\left(\frac{1-\tilde{\lambda}}{1+\widetilde{\lambda}}\right)\left(\frac{1+\widetilde{a}_{i}^{2}}{1-\widetilde{a}_{i}^{2}}\right) \widetilde{a}_{i} \frac{d r_{h}}{r_{h}}\right|_{\Omega, \lambda}
$$


and from (96)

$$
\left.\frac{d \Xi_{i}}{\Xi_{i}}\right|_{\Omega, \lambda}=-\left.\frac{4 \widetilde{\lambda} \widetilde{a}_{i}^{2}}{(1+\widetilde{\lambda})\left(1-\widetilde{a}_{i}^{2}\right)} \frac{d r_{h}}{r_{h}}\right|_{\Omega, \lambda} .
$$

Using these it is straightforward to show that

$$
\left.\frac{\partial T}{\partial r_{h}}\right|_{\Omega, \lambda}=-\frac{1}{4 \pi r_{h}^{2}}\left\{D-3+2 \bar{\Sigma}_{1}-\tilde{\lambda}\left(D-1+2 \bar{\Sigma}_{1}\right)\right\},
$$

where $\bar{\Sigma}_{1}:=\sum_{i=1}^{N} \frac{\widetilde{a}_{i}^{2}}{1-\widetilde{a}_{i}^{2}}$, and

$$
\left.\frac{\partial S}{\partial r_{h}}\right|_{\Omega, \lambda}=\frac{S}{r_{h}}\left(D-2+2 \bar{\Sigma}_{1}\right) .
$$

Combining these we immediately arrive at equation (47) in the text,

$$
C_{\Omega}=-\frac{4 \pi r_{h} T S\left(D-2+2 \bar{\Sigma}_{1}\right)}{D-3+2 \bar{\Sigma}_{1}-\widetilde{\lambda}\left(D-1+2 \bar{\Sigma}_{1}\right)} .
$$

This generalises the asymptotically anti-de Sitter $D=4$ case derived in [7] to arbitrary $D$. It also generalises the asymptotically flat $D$-dimensional case derived in [5] to asymptotically AdS.

\section{Isentropic moment of inertia}

To calculate the isentropic moment of inertia we can first use (97) to obtain

$$
\begin{aligned}
\left.d r_{h}\right|_{S, \lambda} & =-\left.\frac{2 r_{h}(1+\widetilde{\lambda})}{(D-2+2 \widetilde{\lambda} X)} \sum_{j=1}^{N} \frac{\widetilde{a}_{j} d \widetilde{a}_{j}}{\left(1+\widetilde{a}_{j}^{2}\right) \Xi_{j}}\right|_{S, \lambda} \\
\left.\Rightarrow \quad \frac{1}{r_{h}} \frac{\partial r_{h}}{\partial \widetilde{a}_{j}}\right|_{S, \lambda} & =-\frac{2(1+\widetilde{\lambda})}{(D-2+2 \widetilde{\lambda} X)} \frac{\widetilde{a}_{j}}{\left(1+\widetilde{a}_{j}^{2}\right) \Xi_{j}}
\end{aligned}
$$

with $X:=\sum_{k=1}^{N} \frac{\widetilde{a}_{k}^{2}}{\Xi_{k}}$. Then

$$
\Omega_{i}=\frac{\widetilde{a}_{i}}{r_{h}\left(1+\widetilde{a}_{i}^{2}\right)}
$$


results in

$$
\left.\frac{\partial \Omega_{i}}{\partial \widetilde{a}_{j}}\right|_{S, \lambda}=\frac{(1+\widetilde{\lambda})}{r_{h}\left(1+\widetilde{a}_{i}^{2}\right)\left(1+\widetilde{a}_{j}^{2}\right)}\left[\left(1-\widetilde{a}_{i}^{2}\right) \delta_{i j}+2 \frac{\left(1-\widetilde{\lambda}_{)} \widetilde{a}_{i} \widetilde{a}_{j}\right.}{(D-2+2 \widetilde{\lambda} X) \Xi_{j}}\right] .
$$

This can be inverted as a matrix to give

$$
\left.\frac{\partial \widetilde{a}_{i}}{\partial \Omega_{k}}\right|_{S, \lambda}=\frac{r_{h}}{(1+\widetilde{\lambda})} \frac{\left(1+\widetilde{a}_{i}^{2}\right)\left(1+\widetilde{a}_{k}^{2}\right)}{\left(1-\widetilde{a}_{i}^{2}\right)\left(1-\widetilde{a}_{k}^{2}\right)}\left[\left(1-\widetilde{a}_{i}^{2}\right) \delta_{i k}-2 \frac{(1-\tilde{\lambda}) \widetilde{a}_{i} \widetilde{a}_{k}}{\left(D-2+2 \bar{\Sigma}_{1}\right) \Xi_{k}}\right]
$$

Furthermore

$$
J_{i}=(1+\widetilde{\lambda}) \frac{S}{2 \pi} \frac{\widetilde{a}_{i}}{\Xi_{i}}
$$

yields

$$
\left.\frac{\partial J_{i}}{\partial \widetilde{a}_{k}}\right|_{S, \lambda}=(1+\widetilde{\lambda}) \frac{S}{2 \pi \Xi_{i}^{2}}\left[\Xi_{i} \delta_{i k}-\frac{4 \widetilde{\lambda}}{(D-2+2 \widetilde{\lambda} X)} \frac{\widetilde{a}_{i} \widetilde{a}_{k}\left(1+\widetilde{a}_{i}^{2}\right)}{\Xi_{k}\left(1+\widetilde{a}_{k}^{2}\right)}\right] .
$$

Equations (109) and (111) can now be combined to give the result quoted in the text,

$$
\mathcal{I}^{i j}=\frac{r_{h} S}{2 \pi} \frac{1}{\Xi_{i} \Xi_{j}} \frac{\left(1+\widetilde{a}_{i}^{2}\right)\left(1+\widetilde{a}_{j}^{2}\right)}{\left(1-\widetilde{a}_{i}^{2}\right)\left(1-\widetilde{a}_{j}^{2}\right)}\left\{\Xi_{i}\left(1-\widetilde{a}_{i}^{2}\right) \delta_{i j}-\frac{2(1+\tilde{\lambda}) \widetilde{a}_{i} \widetilde{a}_{j}}{\left(D-2+\bar{\Sigma}_{1}\right)}\right\} .
$$

This extends the asymptotically flat result in 33 , to $\lambda \neq 0$.

The determinant is

$$
\operatorname{det}(\mathcal{I})=\left(\frac{r_{h} S}{2 \pi}\right)^{N} \frac{(D-2-2 \widetilde{\lambda} \bar{X})}{\left(D-2+2 \bar{\Sigma}_{1}\right)} \prod_{k=1}^{N} \frac{\left(1+\widetilde{a}_{k}^{2}\right)^{2} \bar{\Xi}_{k}}{\left(1-\widetilde{a}_{k}\right) \Xi_{k}^{2}}
$$

where

$$
\bar{X}:=\sum_{k=1}^{N} \frac{\widetilde{a}_{k}^{2}}{\bar{\Xi}_{k}}
$$

Finally we note that there is a cancellation of factors in the combination $\beta C_{\Omega} \operatorname{det}(\mathcal{I})=-8 \pi^{2}\left(\frac{r_{h} S}{2 \pi}\right)^{N+1} \frac{(D-2-2 \widetilde{\lambda} \bar{X})}{\left[D-3+2 \bar{\Sigma}_{1}-\widetilde{\lambda}\left(D-1+2 \bar{\Sigma}_{1}\right)\right]} \prod_{k=1}^{N} \frac{\left(1+\widetilde{a}_{k}^{2}\right)^{2} \bar{\Xi}_{k}}{\left(1-\widetilde{a}_{k}\right) \Xi_{k}^{2}}$. 


\section{References}

[1] S.W. Hawking, Nature 248 (1974) 30;

S.W. Hawking, Comm. Math. Phys. 43 (1975) 199;

S.W. Hawking, Phys. Rev. D13 (1976) 191.

[2] S.W. Hawking and D.N. Page, Comm. Math. Phys. 87 (1983) 577.

[3] J.W. York Jr., Phys. Rev. D 33 (1986) 2092.

[4] O.J.C. Dias, P. Figueras, R. Monteiro, H.S. Reall and J.E. Santos, JHEP 1005 (2010) 076, arXiv:1001.4527 [hep-th]].

[5] B.P. Dolan, On the thermodynamic stability of rotating black holes in higher dimensions - a comparison of thermodynamic ensembles, [arXiv1312.6810].

[6] J.M. Maldecena, Adv. Theor. Math. Phys. 2 (1998) 231, arXiv:hep-th/9711200];

S.S. Gubser, I.R. Klebanov and A.M. Polyakov, Phys. Lett. B 428 (1998) 105, [hep-th/9802109];

E. Witten, Adv. Theor. Math. Phys. 2 (1998) 253, [hep-th/9802150].

[7] R. Monteiro, M.J. Perry and J.E. Santos Phys. Rev. D81 (2010) 024001, arXiv:0905.2334.

[8] R. Monteiro, Classical and thermodynamic stability of black holes, University of Cambridge PhD thesis, arXiv:1006.5358.

[9] O.J.C. Dias, P. Figueras, R. Monteiro and J.E. Santos, JHEP 1012 (2010) 067, arXiv:1011.0996.

[10] D. Kastor, S. Ray and J. Traschen, Class. Quantum Grav. 26 (2009) 195011, arXiv:0904.2765].

[11] S. A. Hayward, Class. Quantum Grav. 15, (1998) 3147, gr-qc/9710089].

[12] M. K. Parikh, Phys. Rev. D73, (2006) 124021, [hep-th/0508108].

[13] W. Ballik and K. Lake, The volume of stationary black holes and the meaning of the surface gravity, arXiv:1005.1116 [gr-qc]]. 
[14] W. Ballik and K. Lake, Phys. Rev. D88, (2013) 104038, arXiv:1310.1935 [gr-qc]].

[15] B.P. Dolan, Class. Quantum Grav. 28 (2011) 125020, arXiv:1008.5023].

[16] N. Altamirano, D. Kubizn̆äk, R. B. Mann and Z. Sherkatghanad, Galaxies 2, (2014) 89, arXiv:1401.2586 [hep-th]].

[17] H. Callen, Thermodynamics, (1960) Wiley.

[18] G.W. Gibbons, M.J. Perry and C.N. Pope, Class. Quantum Grav. 22 (2005) 1503, hep-th/0408217.

[19] L. Smarr, Phys. Rev. Lett. 30 (1973) 71.

[20] G. Ruppeiner, Thermodynamic curvature and black holes, arXiv:1309.0901.

[21] A. Chamblin, R. Emperan. C.J. Johnson and R. Myers, Phys. Rev. D60 (1999) 064018, hep-th/9902170]; Phys. Rev. D60 (1999) 104026, hep-th/9904197].

[22] M.M. Caldarelli, C. Cognola and D. Klemm, Class. Quantum Grav. 17 (2000) 299, [hep-th/9908022].

[23] D.Kubizn̆ák and R.B. Mann, JHEP 1207 (2012) 033, arXiv:1205.0559].

[24] B.P. Dolan, Where is the PdV term in the first law of black hole thermodynamics?, Open Questions in Cosmology, ed. G.J. Olmo (2012) InTech (ISBN 978-953-51-0880-1), arXiv:1209.1272.

[25] N. Altamirano, D. Kubizn̆ák, R.B. Mann and Z. Sherkatghanad, KerrAdS analogue of triple point and solid/liquid/gas phase transition, arXiv:1308.2672].

[26] N. Altamirano, D. Kubizn̆ák and R.B. Mann, Phys. Rev. D88 (2013) 101502, arXiv:1306.5756.

[27] R.C. Myers and M.J. Perry, Ann. Phys. 172 (1986) 304.

[28] S.W. Hawking, C.J. Hunter and M.M. Taylor-Robinson, Phys. Rev. D59 (1999) 064005, hep-th/9811056. 
[29] G.W. Gibbons, H. Lü, D.N. Page and C.N. Pope, Geom. Phys. 53 (2005) 49, hep-th/0404008;

Phys. Rev. Lett. 93 (2004) 171102, |hep-th/0409155|.

[30] B.P. Dolan, Class. Quantum Grav. 28 (2011) 235017, arXiv:1106.6260.

[31] M. Cvetic, G.W. Gibbons, D. Kubizn̆ák and C.N. Pope, Phys. Rev. D84 (2011) 024037, arXiv:1012.2888.

[32] B.P. Dolan, D. Kastor, D. Kubizn̆ák, R.B. Mann and J. Traschen, Phys. Rev. D87 (2103) 104017, arXiv:1301.5926].

[33] B.P. Dolan, Class. Quantum Grav. 31 (2014) 035022, arXiv:1308.5403)].

[34] C. Niu, Y. Tian and X. Wu, Phys. Rev. D85 (2012) 024017, arXiv:1104.3066 [hep-th]].

[35] V. Cardoso and Ó.J.C. Dias, Phys. Rev. D70 (2004) 084011, arXiv:hep-th/0405006.

[36] V. Cardoso, Ó.J.C. Dias and S. Yoshida, Phys. Rev. D74 (2006) 044008, arXiv:hep-th/0607162.

[37] M. Shibata and H. Yoshino, Phys. Rev. D81 (2010) 021501, arXiv:0912.3606].

[38] H. Elvang and P.Figueras, JHEP 0705 (2007) 050, hep-th/0701035.

[39] H. Elvang, R. Emparan and P. Figueras, JHEP 0705 (2007) 056, arXiv:hep-th/0702111.

[40] R. Emparan, T. Harmark, V. Niarchos, N.A. Obers and M.J. Rodriguez, JHEP 0710 (2007) 110, arXiv:0708.2181.

[41] M.M. Caldarelli, R. Emparan and M.J. Rodriguez, JHEP 0811 (2008) 011, arXiv:0806.1954.

[42] R. Emparan and P. Figueras, JHEP 1011 (2010) 022, arXiv:1008.3243].

[43] H. Iguchi and T. Mishima, Phys. Rev. D82 (2010) 084009, arXiv:1008.4290].

[44] J. Armas and N.A. Obers, Phys. Rev. D83 (2011) 084039, arXiv:1012.5081]. 